\title{
UTILIZACIÓN DE TOXINA BOTULÍNICA EN EL MANEJO DE ONFALOCELE GIGANTE EN UN LACTANTE. INFORME DE CASO
}

\section{USE OF BOTULINUM TOXIN IN THE MANAGEMENT OF GIANT OMPHALOCELE IN AN INFANT. CASE REPORT}

\author{
Juan Jose Salinas Barreto ${ }^{1 a}$, Felix Fernando Rosas Ayque ${ }^{1 a}$.
}

\begin{abstract}
RESUMEN
Introducción: El onfalocele gigante se define por defecto de pared abdominal de $5 \mathrm{~cm}$ de diámetro a más o presencia de herniación hepática. Se ha descrito el uso de toxina botulínica como coadyuvante en la reconstrucción de la pared abdominal en adultos, su utilidad en pediatría aun es poco descrito, pero si muestra seguridad y eficacia.

Reporte de Caso: Paciente femenino de 40 semanas de edad gestacional con onfalocele gigante con defecto amplio de aproximadamente 9 centímetros, en quien a los 75 días de vida se le aplica inyecciones de toxina botulínica alrededor del defecto y es intervenida a la semana para al cierre definitivo de pared abdominal, la cual se logra sin tensión ni intercurrencias post operatorias. Conclusión: El uso de toxina botulínica muestra tener alta efectividad para lograr un cierre de pared abdominal en pacientes con onfalocele gigante.
\end{abstract}

Palabras clave: Hernia umbilical, toxina botulínica, lactante. (Fuente: DeCS BIREME).

\begin{abstract}
Introduction: Giant omphalocele is an abdominal wall defect of $5 \mathrm{~cm}$ in diameter or more or presence of liver herniation. The use of botulinum toxin has been described as an adjunct therapy in the reconstruction of the abdominal wall in adults. Its use in pediatric patients is still little described but it does show safety and efficacy.

Case Report: 40-week gestational-age female patient with a giant omphalocele with a wide defect measuring approximately 9 centimeters, in whom at 75 days of life botulinum toxin injections were applied around the defect and operated a week after for definitive abdominal wall closure which was achieved without tension or post-operative intercurrences. Conclusion: The use of botulinum toxin is shown to be highly effective in achieving abdominal wall closure in infant patients with giant omphalocele.
\end{abstract}

Keywords: Hernia umbilical, botulinum toxins, infant. (Source: MeSH NLM).

\section{INTRODUCCIÓN}

El onfalocele es un defecto congénito de la pared abdominal presente en 1 de 40000 a 6000 nacidos vivos $^{1}$. El onfalocele gigante se define por un defecto de pared abdominal de $5 \mathrm{~cm}$ de diámetro a más o presencia de herniación hepática². El manejo del onfalocele gigante es un desafío hasta la actualidad teniendo como opciones un cierre quirúrgico por etapas en la cual el cierre de la pared abdominal se logra tras múltiples cirugías y manejo conservador en el cual se busca la epitelización de las membranas amnióticas y un cierre quirúrgico posterior ${ }^{1}$
Se ha descrito el uso de toxina botulínica como coadyuvante en la reconstrucción de la pared abdominal en adultos ${ }^{3}$, su utilidad en el uso de defectos de pared abdominal en lactantes y niños es poco descrito. Pero si se usa con seguridad en otras patologías pediátricas como hiperhidrosis, migraña, labio leporino, hipertrofias musculares entre otras ${ }^{4}$. La toxina botulínica actúa inhibiendo la liberación de acetilcolina que previene la contracción muscular, tiene un efecto que puede durar hasta los 6 meses de su aplicación aunque el pico máximo de acción ocurre alrededor de los 14 días de aplicación. Además, se ha evidenciado un efecto antiinflamatorio y disminuye el efecto cicatricial debido a inhibición en la liberación de glutamato y sustancia $\mathrm{B}^{4}$.

\footnotetext{
Servicio de Cirugía Neonatal, Instituto Nacional Materno Perinatal, Lima-Perú.

a Cirujano Pediatra.
}

iD Código ORCID: https://orcid.org/0000-0002-5519-9470: Juan Jose Salinas Barreto

Citar como: Salinas Barreto JJ, Rosas Ayque FF. Utilización de toxina botulínica en el manejo de onfalocele gigante en un lactante. Informe de caso. Rev Peru Investig Matern Perinat. 2021;10(2): 56-59

DOI https://doi.org/10.33421/inmp.20212231 


\section{REPORTE DE CASO}

Paciente femenino con antecedente de defecto de pared abdominal: onfalocele gigante en ecografía prenatal, nacido por cesárea electiva a las 40semanas de edad gestacional con un peso de $4080 \mathrm{gr}$ macrosómico y un Apgar de $8-9$.

Evaluado a las 3 horas de nacido por cirugía pediátrica donde se encuentra paciente despierto activo, reactivo, ventilando espontáneamente con defecto de pared central de aproximadamente 8 a 9 $\mathrm{cm}$ de diámetro, cubierto por membranas amnióticas integras con protrusión de hígado predominantemente $(80 \%)$ y asas intestinales delgadas, se decide manejo conservador con aplicación de rifamicina spray y vendaje compresivo para epitelización del defecto y buscar introducción de contenido herniado a cavidad abdominal y se evaluara cierre quirúrgico del defecto aproximadamente a los dos meses de nacido. Se indica nutrición enteral total ya que no había signos de obstrucción intestinal.

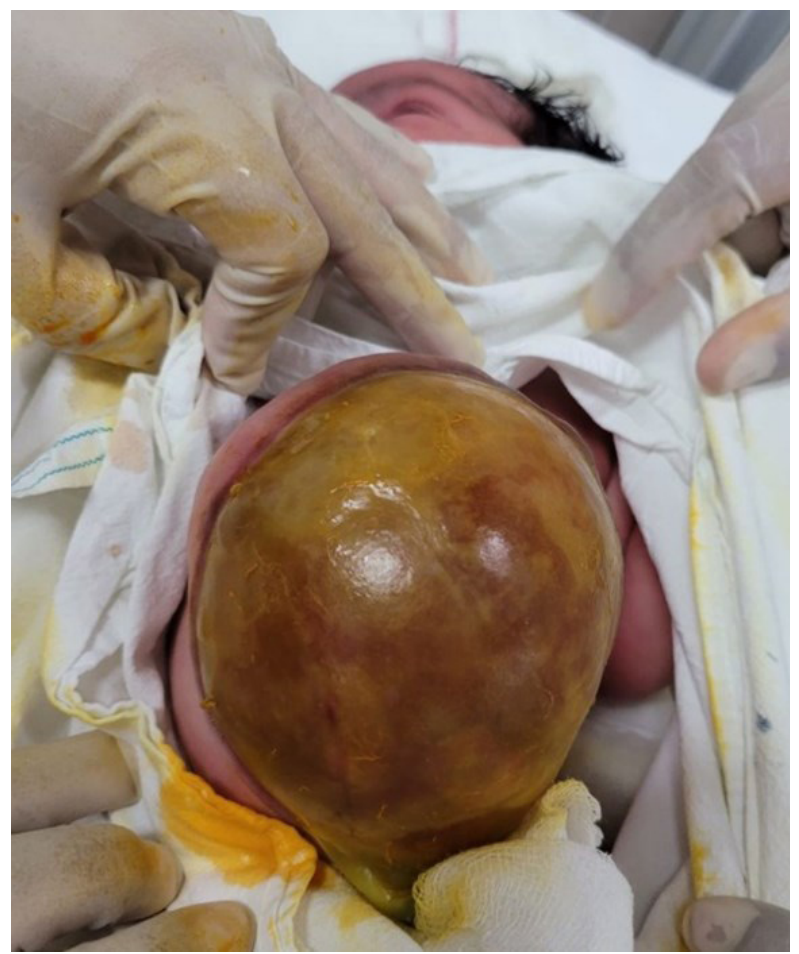

Figura 1. Onfalocele gigante en primer día de vida

La hospitalización a los dos meses ocurre sin intercurrencias las membranas del onfalocele epitelizan aproximadamente un $80 \%$ y se logra reducir el contenido protruido considerablemente pero el defecto de pared persiste amplio de 9 centímetros aproximadamente y con herniación de hígado importante. Por lo que se decide utilizar inyecciones intramusculares de toxina botulínica alrededor del defecto de pared abdominal. Se toma la decisión de realizar el procedimiento en sala de operaciones, se toma prueba molecular PCR para SARS cov-2 previo a procedimiento con resultado positivo pasando a aislamiento por dos semanas, al culminar aislamiento, con prueba molecular PCR para SARS cov-2 negativa y a los 75 días de vida de la paciente, se procede a colocar las inyecciones de toxina botulínica en sala de operaciones con anestesia general en ventilación espontanea con ketamina y propofol endovenoso. Las inyecciones se colocan alrededor del defecto en 10 puntos alrededor de su circunferencia, 5 aplicaciones para hemiabdomen izquierdo y 5 para el hemiabdomen derecho, en cada inyección se aplicaron 2.5 unidades de toxina botulínica, dando un total de 25 unidades aplicadas al paciente. Procedimiento sin intercurrencias regresando a la unidad de cuidados intermedios para monitorización.

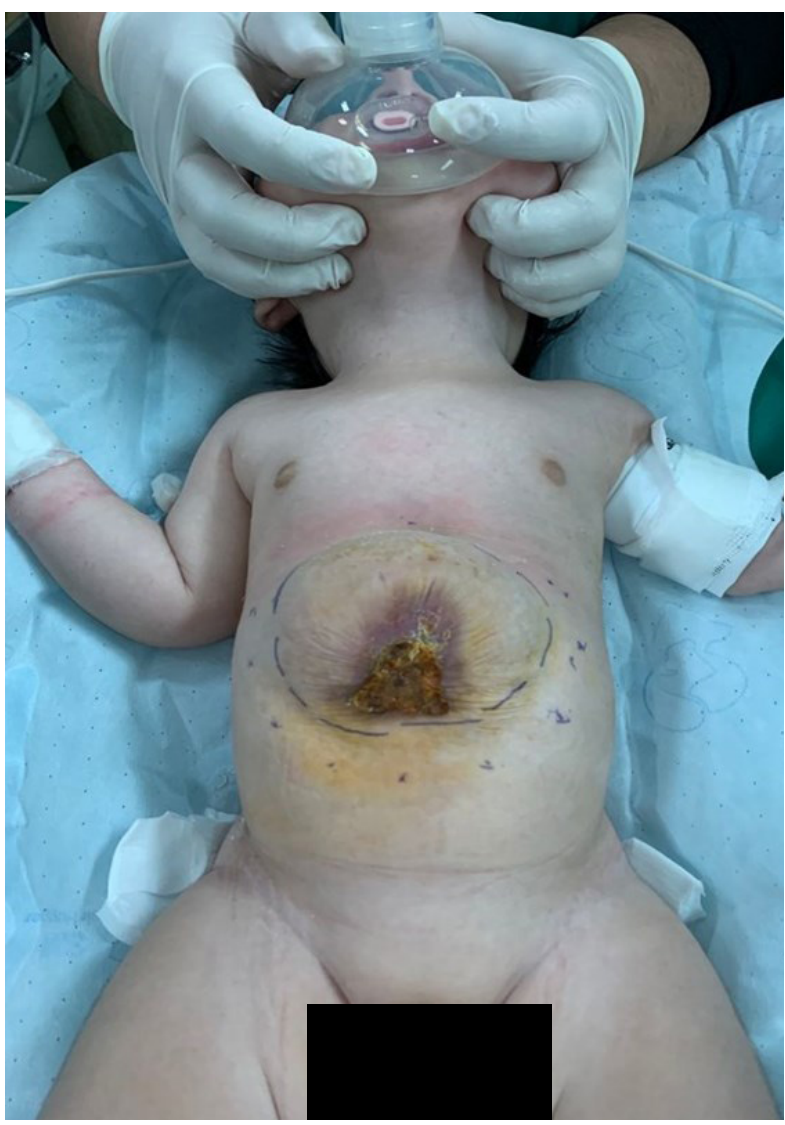

Figura 2. Reparos para la aplicación de toxina botulínica

A la semana del procedimiento paciente ingresa a sala de operaciones para realizar cierre definitivo de la pared abdominal el cual se logra realizar con una sutura mediana longitudinal con puntos separados sin tensión ni aumento de la presión intraabdominal, permitiendo que la paciente sea extubada al finalizar la cirugía y regrese a la unidad de cuidados intermedios para vigilancia y manejo del dolor. 


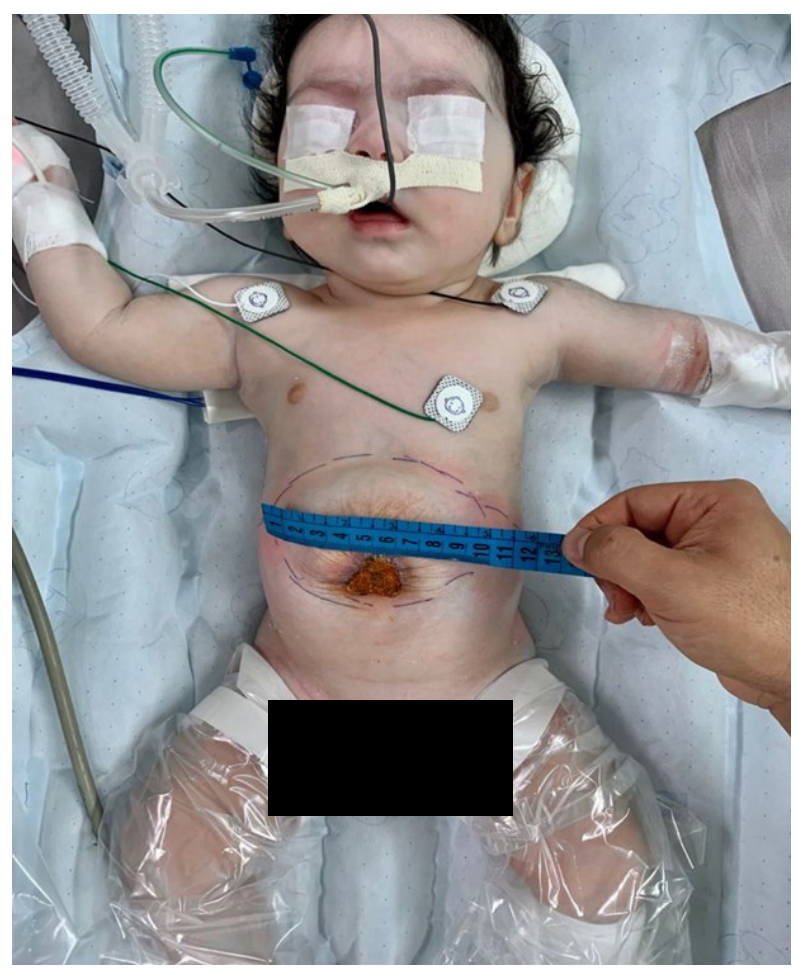

Figura 3. Medición del defecto de pared abdominal previo a la cirugía

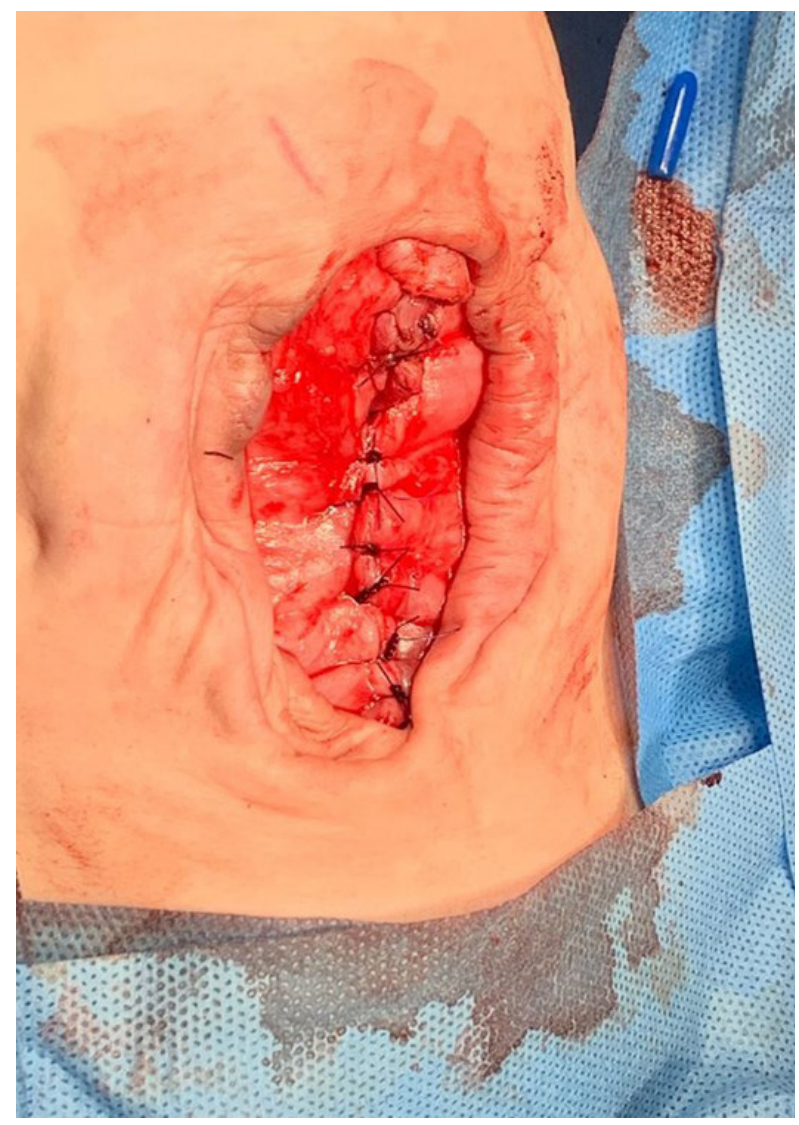

Figura 4. Cierre de pared abdominal: aponeurosis musculo.

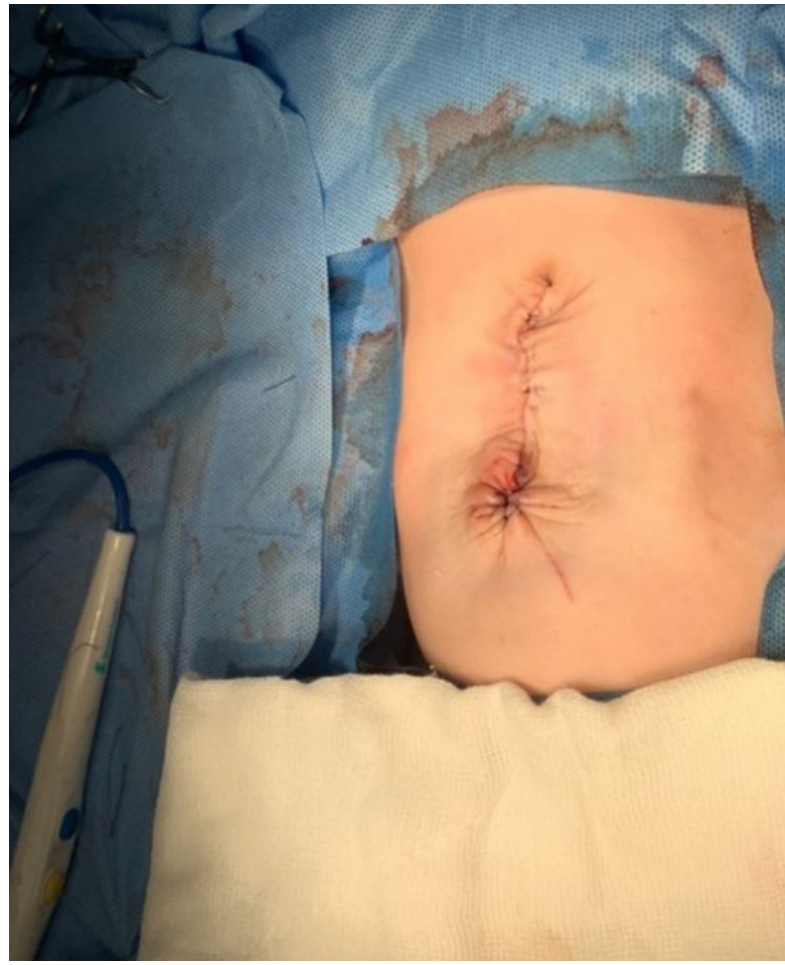

Figura 5. cierre total del defecto de pared abdominal.

En su primer día post operatorio paciente inicia nutrición enteral con lactancia materna alcanzando el volumen total al cuarto día post operatorio y se indica el alta domiciliaria al quinto día de la cirugía. A la fecha de publicación la paciente no presenta ninguna complicación ni intercurrencias con una evolución favorable.

\section{DISCUSIÓN}

El manejo del onfalocele gigante sigue siendo controversial, en nuestra institución se maneja de manera conservadora, se busca la epitelización del defecto con aplicaciones tópicas de rifamicina y se realiza vendaje compresivo, técnica de pintar y esperar ${ }^{5}$, operándose cerca de los dos meses para lograr un cierre definitivo de la pared abdominal. En este caso particular debido al defecto tan amplio, se decidió aplicar toxina botulínica ya que no se han reportado efectos adversos ${ }^{6}$.

Debido a sus efectos la aplicación incrementa la laxitud de la pared abdominal ${ }^{7}$, disminuye su grosor permitiendo un ensanchamiento de la cavidad abdominal que facilitaría el cierre primario evitando el uso de mallas y disminuye el riesgo de presentar un síndrome compartimental, las complicaciones respiratorias o ventilatorias producto de un cierre con tensión intraabdominal incrementada y favorece a su vez el manejo post operatorio del dolor ${ }^{8-13}$. 
La dosis pediátrica recomendada por la FDA propone $8 \mathrm{UI} / \mathrm{kg}$ hasta un máximo de 300 unidades $^{14}$. Aunque la mayoría de revisiones describe la utilización de 2 - $5 \mathrm{UI} / \mathrm{kg}$ decidimos utilizar la dosis de $5 \mathrm{UI} / \mathrm{kg}$ según la recomendación de Eyer de Jesus et $\mathrm{al}^{15}$. No existe una forma específica descrita de cómo realizar las aplicaciones de toxina botulínica en estos pacientes, la literatura reporta entre 3 a 5 inyecciones por lado para tratar esta patología, Eyer de Jesus et al realiza una comparación con las publicaciones reportada hasta el $2020^{15}$. En nuestro caso se utilizaron 5 inyecciones por lado para abarcar un mayor una mayor área de pared abdominal con el efecto de la toxina, la literatura describe las aplicaciones de inyecciones bajo guía ecográfica, en nuestra institución no contamos con ecografía a disponibilidad por lo que se aplicó sin guía ecográfica al paciente en sala de operaciones bajo anestesia general. Está descrito que el efecto máximo que se alcanza con la toxina botulínica ocurre alrededor de las dos semanas sin embargo pudimos evidenciar que a la semana hubo un agrandamiento de la cavidad y un aumento de la laxitud muscular por lo que se decidió el ingreso a sala de operaciones para el cierre de la pared abdominal.

La utilización de toxina botulínica en pacientes pediátricos con defectos de pared está aún poco reportada, pero demuestra ser segura permitiendo realizar un cierre de la pared abdominal sin necesidad de utilizar material protésico o reintervenciones.

En conclusión, el uso de toxina botulínica pre operatoria para pacientes lactantes con diagnóstico de onfalocele gigante muestra tener alta efectividad para lograr un cierre de pared abdominal sin necesidad de utilizar material protésico o de reintervenciones, la técnica de aplicación es segura y fácilmente reproducible y muestra una nueva alternativa en el manejo de esto pacientes.

Consentimiento informado: Se cuenta con autorización de la madre de la lactante para la publicación de este reporte.

Conlicto de intereses: El autor declara no tener conflicto de intereses en la publicación de este reporte.

Aprobacion por comisión de ética: Se cuenta con la aprobación del comité de ética de la institución para la publicación de este reporte.

Financiamiento: Autofinanciado.

Conflictos de interés: Los autores declaran no tener algún conflicto de intereses.

\section{REFERENCIAS BIBLIOGRÁFICAS}

1. deVries PA. The pathogenesis of gastroschisis and omphalocele. J Pediatr Surg 1980 ; 15 ( 3 ) : 245 - 51

2. Whitehouse JS, et al . Conservative management of giant omphalocele with topical povidone - iodine and its effect on thyroid function . J Pediatr Surg 2010; 45 (6):1192-7.

3. Steven J. Capece, Sean J. Wallace, Randolph Wojcik, Marybeth Brownece. Botulinum Toxin for giant omphalocele abdominal wall reconstruction. Case report J Pediatr Surg 2020;61:101562.

4. Fu K, Teichgraeber J, Greives M. Botulinum toxin use in pediatric plastic surgery. Ann Plast Surg 2016;77:57782.

5. Wagner J, Cusick R. Paint and wait management of giant omphaloceles. Semin Pediatr Surg 2019;28:95-100.

6. Jessica L. Rauh, Leah M. Sieren, Giant omphalocele closure utilizing botulinum toxin. Case report J Pediatr Surg 2020;60 : 101534.

7. Motz B, Schlosser K, Heniford B. Chemical components separation. Plast Reconstr Surg 2018;142:58S-63S.

8. Zendejas B, Khasawneh MA, Srvantstyan B, Jenkins DH, Schiller HJ, Zielinski MD. Outcomes of chemical component paralysis using botulinum toxin for incisional hernia repairs. World J Surg. 2013;37:2830-7.

9. Elstner KE, Read JW, Saunders J, Cosman PH, Rodriguex-Acevedo $\mathrm{O}$, et al. Selective muscle botulinum toxin $\mathrm{A}$ component paralysis in complex ventral hernia repair. Hernia. https://doi.org/10.1007/s10029-019-01939-3

10. Ibarra-Hurtado TR, Nuno-Guzman CM, Echeagaray-Herrera JE, Robles-Velez E,Gonzalez-Jaime JJ. Use of botulinum toxin type A before abdominal wall hernia reconstruction. World J Surg. 2009;33:2553-6.

11. Bueno-Lledo J, Gallud AT, Rosellon RJ, Tatay FC, Pastor PG, Diana SB, Hernandez JI. Preparacion preoperatoria de la hernia con perdida de domicilio. Neumoperitoneo progresivo y toxina botulinica do tipo A. Cir Esp. 2017:245-53.

12. Farooque F, Jacombs AS, Roussos E, Read JW, Dardano AN, Edye M, Ibrahim N. Preoperative abdominal muscle elongation with botulinum toxin A for complex incisional ventral hernia repair. ANZ J Surg. 2016;86:79-83.

13. Smoot D, Zielinski M, Jenkins D, Schiller H. Botox A injectionfor pain after laparoscopic ventral hernia. A case report. Pain Medicine. 2011;12:1121-3.

14. Food and Drug Administration: botulinum toxin instructions https://www.accessdata.fda.gov/drugsatfda_docs/label/2019/103000s5309lbl.pdf

15. de Jesus LE, Leve TC, Fulgencio $C$, et al. Botulinum toxin abdominal wall injection and post-omphalocele ventral hernia repair: database and proposal of a protocol. Ann Pediat Surg 16, 56 (2020). https://doi.org/10.1186/s43159-02000058-4

\section{Correspondencia:}

Juan Jose Salinas Barreo

Dirección: Madrid 100 Pueblo Libre

E-mail: juankmed26@gmail.com

Teléfono: 945558094 